\title{
Statistical Model of the Early Stage of nucleus-nucleus collisions with exact strangeness conservation
}

\author{
R. V. Poberezhnyuk, ${ }^{1}$ M. Gazdzicki, ${ }^{2,3}$ and M. I. Gorenstein ${ }^{1,4}$ \\ ${ }^{1}$ Bogolyubov Institute for Theoretical Physics, Kiev, Ukraine \\ ${ }^{2}$ Goethe-University, Frankfurt, Germany \\ ${ }^{3}$ Jan Kochanowski University, Kielce, Poland \\ ${ }^{4}$ Frankfurt Institute for Advanced Studies, Frankfurt, Germany
}

\begin{abstract}
The Statistical Model of the Early Stage, SMES, describes a transition between confined and deconfined phases of strongly interacting matter created in nucleus-nucleus collisions. The model was formulated in the late 1990s for central $\mathrm{Pb}+\mathrm{Pb}$ collisions at the CERN SPS energies. It predicted several signals of the transition (onset of deconfinement) which were later observed by the NA49 experiment. The grand canonical ensemble was used to calculate entropy and strangeness production. This approximation is valid for reactions with mean multiplicities of particles carrying conserved charges being significantly larger than one.

Recent results of NA61/SHINE on hadron production in inelastic $\mathrm{p}+\mathrm{p}$ interactions suggest that the deconfinement may also take place in these reactions. However, in this case mean multiplicity of particles with non-zero strange charge is smaller than one. Thus for the modelling of $p+p$ interactions the exact strangeness conservation has to be implemented in the SMES. This extension of the SMES is presented in the paper.

PACS numbers: 12.40.-y, 12.40.Ee
\end{abstract}

Keywords: Onset of deconfinement, nucleus-nucleus collisions, proton-proton collisions 


\section{INTRODUCTION}

Strongly interacting matter at sufficiently high energy density is predicted to exist in a phase of quasi-free quarks and gluons, the quark gluon plasma (QGP). Relativistic nucleus-nucleus $(\mathrm{A}+\mathrm{A})$ collisions provide a unique opportunity to check this prediction and study properties of the transition to the QGP as well as the QGP itself. This is because the system created in $\mathrm{A}+\mathrm{A}$ collisions is close to (at least local) equilibrium. The conclusion is based on the success of statistical and hydrodynamical models of particle production at high energies (see e.g. Ref. [1]). Consequently, properties of the system (matter) can be characterized by its equation of state which should include different phases and transitions between them. It is important to note that nowadays there is no dynamical understanding of the observed equilibrium properties of particle production in $\mathrm{A}+\mathrm{A}$ collisions.

With increasing collision energy the energy density of matter created at the early stage of $\mathrm{A}+\mathrm{A}$ collisions increases. Thus, at a sufficiently high collision energy the matter is expected to be created in the QGP phase. The beginning of the QGP creation with increasing collision energy is referred to as the onset of deconfinement. The experimental search for the onset of deconfinement in central $\mathrm{Pb}+\mathrm{Pb}$ collisions was performed by the NA49 experiment at the Super Proton Synchrotron (SPS) of the European Organization for Nuclear Research (CERN) about 15 years ago. The study was motivated [2] by predictions of the Statistical Model of the Early Stage (SMES) [3] of A+A collisions. According to the model the onset of deconfinement in central $\mathrm{A}+\mathrm{A}$ collisions should lead to rapid changes of the energy dependence of several hadron production properties, all located in a common energy domain. In particular, a nonmonotonic dependence of the strangeness to entropy ratio as a function of the collision energy (the horn) was predicted [3] as an important signal of the transition. This and other predictions of the SMES were confirmed by NA49 [4, 5]. Moreover, following results from the Relativistic Heavy Ion Collider at Brookhaven National Laboratory and the Large Hadron Collider (LHC) at CERN agree with the NA49 results and their interpretation (see Ref. [6]). The SMES predictions and the experimental evidence for the onset of deconfinement are presented in recent reviews [7].

The SMES is probably the simplest model of the onset of deconfinement. This leads to 
a number of advantages and disadvantages. In particular, the SMES is frequently criticized for being based on simple assumptions which can not be justified within popular dynamical approaches to $\mathrm{A}+\mathrm{A}$ collisions.

In this paper we concentrate on a single aspect of the SMES which concerns the finite size effects for strange hadron production. The SMES predictions for strangeness production were calculated within the grand canonical ensemble (GCE). This approximation is valid for central $\mathrm{Pb}+\mathrm{Pb}$ collisions at the SPS energies in which mean multiplicity of particles with non-zero strange charge is significantly larger than one. However, this is not the case for inelastic $\mathrm{p}+\mathrm{p}$ interactions at the SPS energies. Here the exact strangeness conservation has to be imposed using the canonical ensemble (CE) [14 17].

Recently, the NA61/SHINE Collaboration at the CERN SPS published results on hadron production in $\mathrm{p}+\mathrm{p}$ interactions [9, 10]. They suggest that in these reactions the strangeness to entropy ratio (experimentally replaced by the $K^{+}$to $\pi^{+}$ratio) also changes rapidly in the SPS energy range, see Fig. 1. However, the ratio and its energy dependence are significantly different from the horn measured in central $\mathrm{Pb}+\mathrm{Pb}$ collisions. Can these results be explained by the onset of deconfinement as modelled by the SMES? The first step towards an answer to this question is taken in this paper by introducing the exact strangeness conservation. In order to allow for a direct comparison with the previously published predictions, the remaining SMES assumptions, parameters and notations are kept unchanged.

The paper is organized as follows. In Sec. II the GCE formulation of the SMES is briefly recapitulated. The exact strangeness conservation is introduced in the SMES in Sec. III and results for $\mathrm{p}+\mathrm{p}$ interactions and collisions of small nuclei are presented. A summary in Sec. IV closes the article.

\section{THE SMES MODEL IN BRIEF}

The SMES model was formulated almost 20 years ago. Its basic assumptions, parameters and results are summarized in this section. Together with the notation used in the original paper they are here kept unchanged as much as possible in order to allow for a direct comparison with the previously published results. 


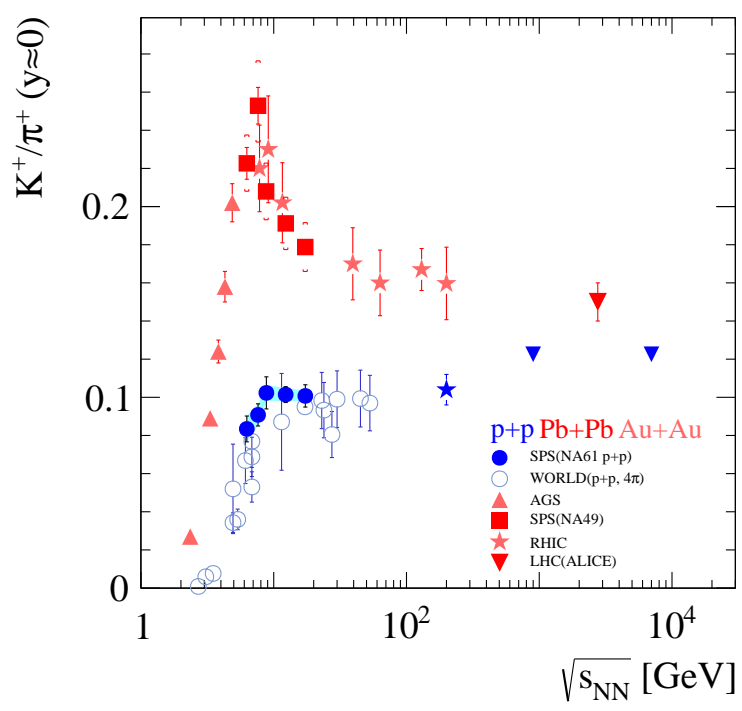

Figure 1: The horn structure in the energy dependence of the $\mathrm{K}^{+} / \pi^{+}$ratio is interpreted as evidence for the onset of deconfinement located at low CERN SPS energies. The structure was first discovered by NA49 in central $\mathrm{Pb}+\mathrm{Pb}$ collisions. Surprisingly its shadow is visible in inelastic $\mathrm{p}+\mathrm{p}$ interactions as indicated by the new NA61/SHINE data.

The SMES assumes that the matter created at the early stage of collisions has zero conserved charges. Consequently, its properties are entirely defined by the available energy and the volume in which production takes place. In central $\mathrm{A}+\mathrm{A}$ collisions this volume is chosen as the Lorentz contracted volume occupied by the colliding nucleons (participant nucleons) from a single nucleus:

$$
V=\frac{4 \pi r_{0}^{3} A_{p} / 3}{\sqrt{s_{N N}} / 2 m_{N}},
$$

where $m_{N}$ is the nucleon mass, $\sqrt{s_{N N}}$ is the center of mass energy of the nucleon pair, $A_{p}$ is the number of participant nucleons from a single nucleus. The $r_{0}$ parameter is taken to be $1.30 \mathrm{fm}$ in order to fit the mean baryon density in the nucleus, $\rho_{0}=0.11 \mathrm{fm}^{-3}$.

Only a fraction, $\eta$, of the total energy in A+A collisions is transformed into the energy of new degrees of freedom created at the early stage. This is because a part of the energy is carried by the net baryon number. The released (inelastic) energy is expressed as

$$
E=\eta\left(\sqrt{s_{N N}}-2 m_{N}\right) A_{p}
$$


where the parameter $\eta$ is assumed to be independent of the collision energy and the system size. The value of $\eta$ used for numerical calculations is $0.67[3]$.

Assumptions (1) and (2) with $\eta=1$ correspond to the Landau hydrodynamical model [11]. Similarly to this model, in the SMES we do not consider dynamical mechanisms leading to a fast thermalization of the matter. The SMES model postulates that the creation of new particles at the early stage of collision is a statistical process, namely, all microscopic states allowed by conservation laws is equally probable.

The SMES predictions for the pion multiplicities are based on the assumption that the entropy generated at the early stage of collision is (approximately) conserved during the expansion of produced matter. It was indeed observed that the dissipative effects estimated by the ratio of the shear viscosity to the entropy density are small for the strongly interacting matter, especially in a region of the deconfinement transition (see, e.g., [12] and references therein). It should be also noted that particle interactions play rather different role for the equilibrium properties (e.g., the equation of state) and the kinetic coefficients (e.g., the shear viscosity). This is clearly demonstrated by a simple example of the hard balls system [13]. The hard core particle radius $r$ leads to small corrections to the ideal gas equation of state due to the excluded volume effects, but the shear viscosity as it behaves as $\propto r^{-2}$ and thus it is strongly dependent on $r$.

The elementary particles of strong interactions are quarks and gluons. The deconfined state is considered to be composed of $u, d$ and $s$ quarks and the corresponding anti-quarks each with internal number of degrees of freedom equal to 6 (3 color states and 2 spin states). The contribution of $c, b$ and $t$ quarks is neglected due to their large masses. The internal number of degrees of freedom for gluons is 16 ( 8 color states and 2 spin states). The masses of gluons and non-strange (anti)quarks are taken to be 0. The strange (anti)quark mass is taken to be $175 \mathrm{MeV}$ [3]. The properties of equilibrated matter are characterized by an equation of state (EoS). For the case of quarks and gluons the bag model EoS is used [8], i.e., the ideal gas EoS modified by a bag constant $B$. This equilibrium state of quarks and gluons is called the Quark Gluon Plasma or Q state.

The SMES uses an effective parametrization of the confined hadron state, denoted as W state. The non-strange degrees of freedom which dominate the entropy production are taken to 
be massless bosons. Their internal number of degrees of freedom is taken to be 16 i.e., about 3 times lower than the internal number of effective degrees of freedom in the QGP. The mass of strange degrees of freedom is assumed to be $500 \mathrm{MeV}$, equal to the kaon mass. The internal number of strange degrees of freedom is assumed to be 14. For the W-state the ideal gas EoS is selected. Clearly, this description of the confined state should only be treated as an effective parametrization. The numerical values of the parameters are fixed by fitting $\mathrm{A}+\mathrm{A}$ data at the AGS, see for details Ref. [3].

The model assumes that always the maximum entropy state is created at the early stage of $\mathrm{A}+\mathrm{A}$ collisions. In the model with two different states ( $\mathrm{W}$ and $\mathrm{Q}$ ) the form of maximum state changes with the collision energy. The regions in which the equilibrium state is in the form of a pure $\mathrm{W}$ or a pure $\mathrm{Q}$ state, are separated by the region in which both states coexist (the mixed phase). The maximum entropy condition is equivalent to the assumption of the first order phase transitions with the Gibbs criterion for the mixed phase (see Appendix B in Ref. [3]). Namely at a given temperature $T$ the system occupies a pure phase $\mathrm{W}$ or $\mathrm{Q}$ whose pressure is larger, the mixed phase is formed if both pressures are equal $p_{W}=p_{Q}$. The transition temperature between the $\mathrm{W}$ and $\mathrm{Q}$ phases is assumed to be $T_{c}=200 \mathrm{MeV}$.

Using the assumptions and parameters defined above predictions of the SMES can be calculated. The early stage energy density reads:

$$
\varepsilon \equiv \frac{E}{V}=\frac{\eta \rho_{0}\left(\sqrt{s_{N N}}-2 m_{N}\right) \sqrt{s_{N N}}}{2 m_{N}}
$$

The pressure and energy density functions in the W-phase and Q-phase are equal to:

$$
\begin{aligned}
& p_{W}(T)=\frac{\pi^{2} g_{W}}{90} T^{4}+\frac{g_{W}^{s}}{2 \pi^{2}} \int_{0}^{\infty} \frac{d k k^{4}}{3\left(k^{2}+m_{W}^{2}\right)^{1 / 2}} \exp \left[-\frac{\left(k^{2}+m_{W}^{2}\right)^{1 / 2}}{T}\right], \\
& \varepsilon_{W}(T)=\frac{\pi^{2} g_{W}}{30} T^{4}+\frac{g_{W}^{s}}{2 \pi^{2}} \int_{0}^{\infty} d k k^{2}\left(k^{2}+m_{W}^{2}\right)^{1 / 2} \exp \left[-\frac{\left(k^{2}+m_{W}^{2}\right)^{1 / 2}}{T}\right], \\
& p_{Q}(T)=\frac{\pi^{2} g_{Q}}{90} T_{c}^{4}+\frac{g_{Q}^{s}}{2 \pi^{2}} \int_{0}^{\infty} \frac{d k k^{4}}{3\left(k^{2}+m_{Q}^{2}\right)^{1 / 2}} \exp \left[-\frac{\left(k^{2}+m_{Q}^{2}\right)^{1 / 2}}{T}\right]-B, \\
& \varepsilon_{Q}(T)=\frac{\pi^{2} g_{Q}}{30} T^{4}+\frac{g_{Q}^{s}}{2 \pi^{2}} \int_{0}^{\infty} d k k^{2}\left(k^{2}+m_{Q}^{2}\right)^{1 / 2} \exp \left[-\frac{\left(k^{2}+m_{Q}^{2}\right)^{1 / 2}}{T}\right]+B .
\end{aligned}
$$

The strange particle contribution to thermodynamical functions (4-7) are taken within the Boltzmann approximation. This simplification is important for the CE treatment which will be 
discussed in the next section. Note that in Ref. [3] the Fermi distribution with $m_{Q}^{*}=175 \mathrm{MeV}$ was used for the strange quarks. In order to minimize differences to the previous results we choose here a larger value of $m_{Q}=216.5 \mathrm{MeV}$ which leads to the same number of strange quarks at the phase transition temperature $\left(T_{c}=200 \mathrm{MeV}\right)$ :

$$
\int_{0}^{\infty} k^{2} d k \exp \left(-\sqrt{k^{2}+m_{Q}^{2}} / T_{c}\right)=\int_{0}^{\infty} k^{2} d k\left[\exp \left(\sqrt{k^{2}+m_{Q}^{* 2}} / T_{c}\right)+1\right]^{-1} .
$$

Then the bag constant $B=570 \mathrm{MeV} / \mathrm{fm}^{3}$ is calculated using the Gibbs criterion of equal pressures:

$$
p_{W}\left(T_{c}\right)=p_{Q}\left(T_{c}\right)
$$

The entropy densities in the pure phases $(i=\mathrm{W}, \mathrm{Q})$ read:

$$
s_{i}(T)=\frac{p_{i}(T)+\varepsilon_{i}(T)}{T} .
$$

In the mixed phase the $\mathrm{W}$ and $\mathrm{Q}$ phases coexist. The fraction of volume occupied by the $\mathrm{Q}$ phase is denoted as $\xi$. The energy and entropy densities in the mixed phase are

$$
\begin{aligned}
& \varepsilon_{\text {mix }}\left(T_{c}\right)=\xi \varepsilon_{Q}\left(T_{c}\right)+(1-\xi) \varepsilon_{W}\left(T_{c}\right), \\
& s_{\text {mix }}\left(T_{c}\right)=\xi s_{Q}\left(T_{c}\right)+(1-\xi) s_{W}\left(T_{c}\right) .
\end{aligned}
$$

The temperature $T$ and pressure $p$ are shown as a functions of the collision energy in Figs. 2 left and right, respectively. The mixed phase starts at collision energy $\sqrt{s_{N N, 1}}$ and ends at $\sqrt{s_{N N, 2}}$ :

$$
\sqrt{s_{N N, 1}}=7.42 \mathrm{GeV}, \quad \sqrt{s_{N N, 2}}=10.83 \mathrm{GeV} .
$$

The equivalence of the Gibbs criterion and the maximum entropy condition is illustrated in Fig. 3 left, where the ratios $R_{i}=s_{i} / s_{Q}$ are presented for $i=\mathrm{W}$, mix, and $\mathrm{Q}$.

The number density of the sum of strange and anti-strange particles in the GCE can be calculated as 

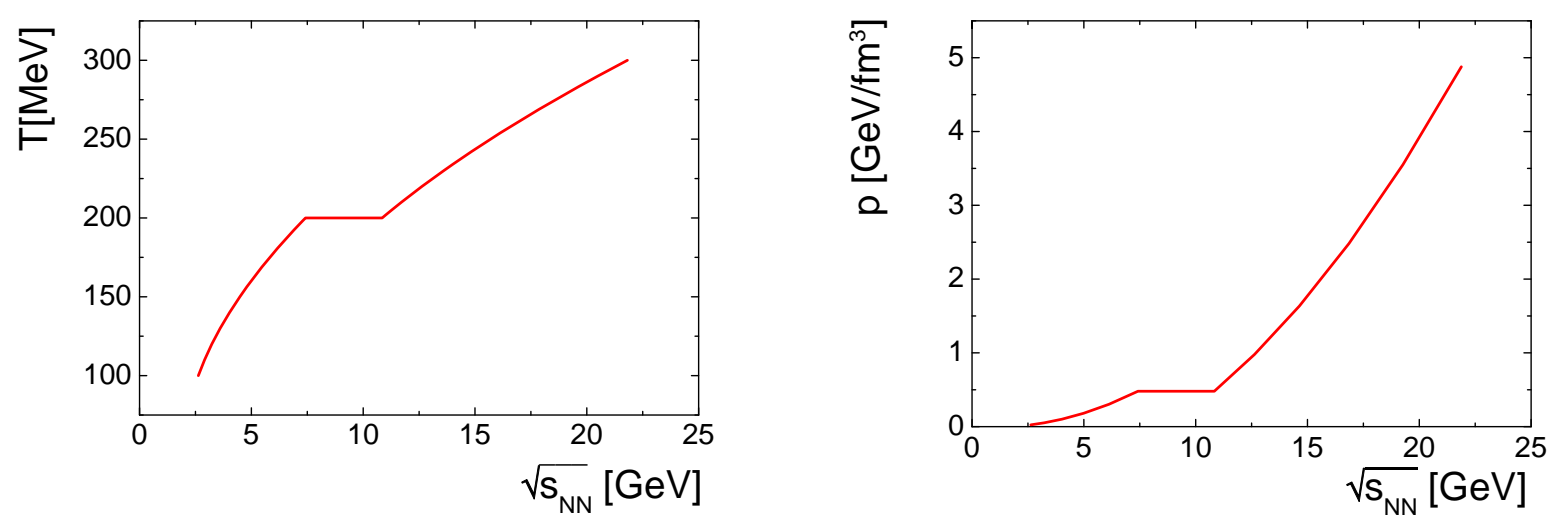

Figure 2: The temperature (left) and pressure (right) of the matter created at the early stage of $\mathrm{A}+\mathrm{A}$ collisions as function of collision energy.
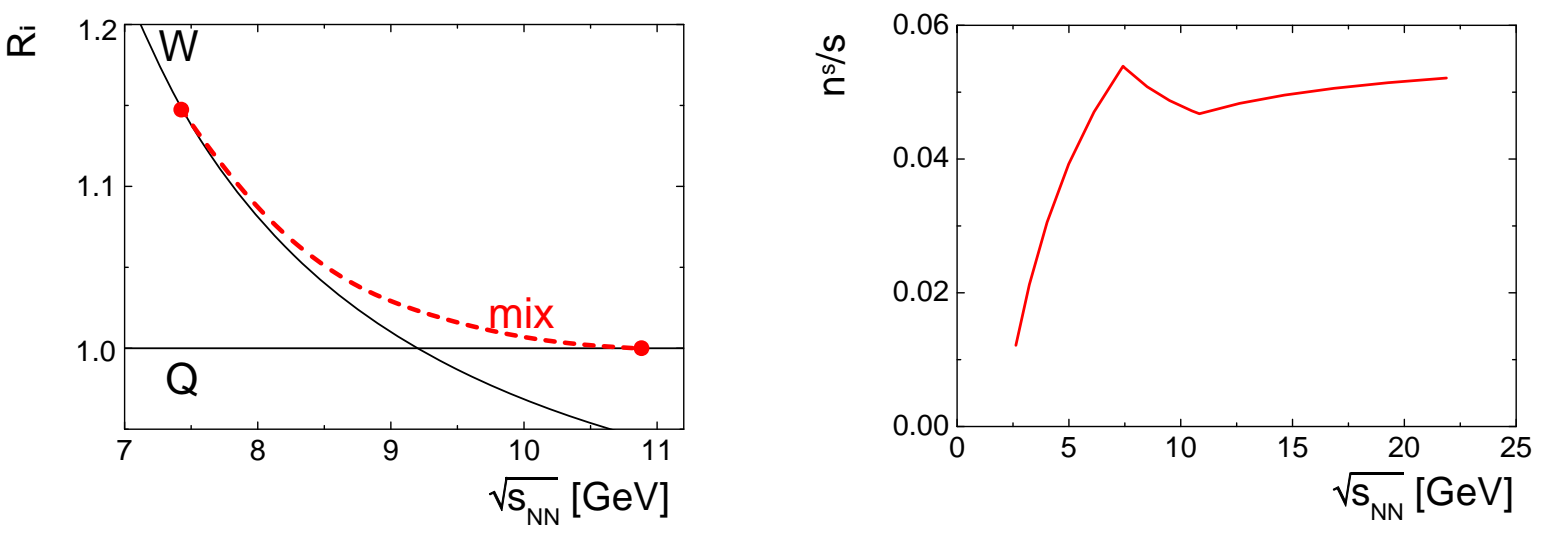

Figure 3: Left: The ratio of entropy densities $s_{i} / s_{Q}$ with $i$ referring to the $\mathrm{W}$ (solid line), Q (horizontal solid line), and mixed (dashed line) phases, as a function of collision energy. The full circles correspond to the beginning and end of the mixed phase given by Eq. (13). Right: The strangeness to entropy ratio $n^{s} / s$ as a function of collision energy. 


$$
\begin{aligned}
& n_{W}^{s}(T)=\frac{g_{W}^{s}}{2 \pi^{2}} \int_{0}^{\infty} d k k^{2} \exp \left[-\frac{\left(k^{2}+m_{W}^{2}\right)^{1 / 2}}{T}\right] \\
& n_{Q}^{s}(T)=\frac{g_{Q}^{s}}{2 \pi^{2}} \int_{0}^{\infty} d k k^{2} \exp \left[-\frac{\left(k^{2}+m_{Q}^{2}\right)^{1 / 2}}{T}\right], \\
& n_{\text {mix }}^{s}(\xi)=\xi n_{Q}^{s}\left(T_{c}\right)+(1-\xi) n_{W}^{s}\left(T_{c}\right) .
\end{aligned}
$$

In Fig. 3 right the strangeness to entropy ratio, $n_{s} / s$, is shown as a function of the collision energy.

\section{PHASE TRANSITION WITH EXACT STRANGENESS CONSERVATION}

In $\mathrm{p}+\mathrm{p}$ interactions at the CERN SPS energies mean multiplicity of produced strange and anti-strange particles is smaller than one. Thus in this case the exact strangeness conservation should be taken into account. In the statistical models this is done within the CE formulation. The CE partition function of strange particles assures an equal number of strange and antistrange charges, $N_{s}=N_{\bar{s}}$, in each microscopic state of the system. For the $\mathrm{W}$ and $\mathrm{Q}$ phases it has a similar form and reads

$$
Z_{c e}(T, V, \lambda)=\sum_{N_{s}=0}^{\infty} \sum_{N_{\bar{s}}=0}^{\infty} \frac{z^{N_{s}}}{N_{s} !} \frac{z^{N_{\bar{s}}}}{N_{\bar{s}} !} \delta\left(N_{s}-N_{\bar{s}}\right)=\frac{1}{2 \pi} \int_{0}^{2 \pi} d \phi \exp \left[z\left(e^{i \phi}+e^{-i \phi}\right)\right]=I_{0}(2 z)
$$

where

$$
z=z_{W, Q}=\lambda \frac{1}{2} V n_{W, Q}^{s}(T)
$$

The auxiliary $\lambda$ parameter in Eq. (18) is introduced to calculate the total strangeness density in the $\mathrm{CE}$ :

$$
n_{W, Q}^{s(C E)}(T, V)=\frac{1}{V}\left[\frac{\partial \ln Z_{c e}}{\partial \lambda}\right]_{\lambda=1}=n_{W, Q}^{s}(T) \frac{I_{1}\left[V n_{W, Q}^{s}(T)\right]}{I_{0}\left[V n_{W, Q}^{s}(T)\right]} .
$$

The ratio of Bessel functions $I_{1}$ and $I_{0}$ in Eq. (19) quantifies the strangeness suppression (relatively to the GCE yield) due to the conservation of net strangeness in each microscopic state of the CE. 
In order to take into account the exact strangeness conservation for thermodynamical functions it is convenient to rewrite them as following:

$$
\begin{aligned}
& p_{W}^{(\mathrm{CE})}(T, V)=\frac{\pi^{2} g_{W}}{90} T^{4}+T n_{W}^{s(\mathrm{CE})}(T, V), \\
& \varepsilon_{W}^{(\mathrm{CE})}(T, V)=\frac{\pi^{2} g_{W}}{30} T^{4}+\omega_{W}(T, V) n_{W}^{s(\mathrm{CE})}(T, V), \\
& p_{Q}^{(\mathrm{CE})}(T, V)=\frac{\pi^{2} g_{Q}}{90} T^{4}+T n_{Q}^{s(\mathrm{CE})}(T, V)-B, \\
& \varepsilon_{Q}^{(\mathrm{CE})}(T, V)=\frac{\pi^{2} g_{Q}}{30} T^{4}+\omega_{Q}(T) n_{Q}^{s(\mathrm{CE})}(T, V)+B,
\end{aligned}
$$

where $n_{W, Q}^{s(\mathrm{CE})}(T, V)$ is given by Eq. 19, , and $\omega_{W, Q}(T)$ is average energy of strange particle:

$$
\omega_{W, Q}(T)=\frac{\int_{0}^{\infty} d k k^{2}\left(k^{2}+m_{W, Q}^{2}\right)^{1 / 2} \exp \left[-\left(k^{2}+m_{W, Q}^{2}\right)^{1 / 2} / T\right]}{\int_{0}^{\infty} d k k^{2} \exp \left[-\left(k^{2}+m_{W, Q}^{2}\right)^{1 / 2} / T\right]},
$$

with $m_{W}$ taken in the $\mathrm{W}$ phase, and $m_{Q}$ in the $\mathrm{Q}$ phase. The entropy density is given by Eq. (10).

For $A_{p} \gg 1$ the system volume (1) is large, and $V n_{W, Q}^{s} \gg 1$. Then one finds that $I_{1}\left[V n_{W, Q}^{s}\right] / I_{0}\left[V n_{W, Q}^{s}\right] \rightarrow 1$ and, therefore, $n_{W, Q}^{s(\mathrm{CE})} \rightarrow n_{W, Q}^{s}$. The results for the CE and GCE become equivalent in this thermodynamical limit, and Eqs. (20,23) coincide with Eqs. (4.7).

In the mixed phase, Eq. (19) should be replaced by

$$
n_{W, Q}^{s, \operatorname{mix}}(T, V, \xi)=n_{W, Q}^{s}(T) \frac{I_{1}[X]}{I_{0}[X]},
$$

where

$$
X=X(T, V, \xi)=\xi V n_{Q}^{s}(T)+(1-\xi) V n_{W}^{s}(T)
$$

is the total GCE number of strange and anti-strange particles (both hadrons and quarks) in the mixed phase. This is because the CE condition of zero net strangeness in the mixed phase should be obeyed by the whole system and not by its phases separately.

At each $\sqrt{s_{N N}}$ one calculates $V$ and $\varepsilon$ according to Eqs. (1) and (3), respectively. The CE Eqs. 19 23) are used to obtain results for the pure $\mathrm{W}$ and $\mathrm{Q}$ phases. In the mixed phase, the temperature $T$ and the parameter $\xi$ are obtained by solving the equations:

$$
\begin{aligned}
& \xi \varepsilon_{Q}^{\operatorname{mix}}[T, X]+(1-\xi) \varepsilon_{W}^{\operatorname{mix}}[T, X]=\varepsilon\left(\sqrt{s_{N N}}\right), \\
& p_{Q}^{\operatorname{mix}}[T, X]=p_{W}^{\operatorname{mix}}[T, X]
\end{aligned}
$$


where $\varepsilon_{W, Q}^{\operatorname{mix}}$ and $p_{W, Q}^{\text {mix }}$ are given by Eqs. 20 23, with $n_{W, Q}^{s, \text { mix }} 25$ instead of $n_{W, Q}^{s,(\mathrm{CE})} 19$.

The collision energy $\sqrt{s_{N N, 1}}$ and temperature $T_{1}$, where the mixed phase starts, and $\sqrt{s_{N N, 2}}$ and $T_{2}$, where the mixed phase ends, are obtained as solutions of Eqs. (27) 28 for $\xi=0$ and $\xi=1$, respectively. One finds:

$$
\begin{aligned}
& T_{1}=203.4 \mathrm{MeV}, \quad \sqrt{s_{N N, 1}}=7.20 \mathrm{GeV}, \\
& T_{2}=202.9 \mathrm{MeV}, \quad \sqrt{s_{N N, 2}}=10.75 \mathrm{GeV} .
\end{aligned}
$$
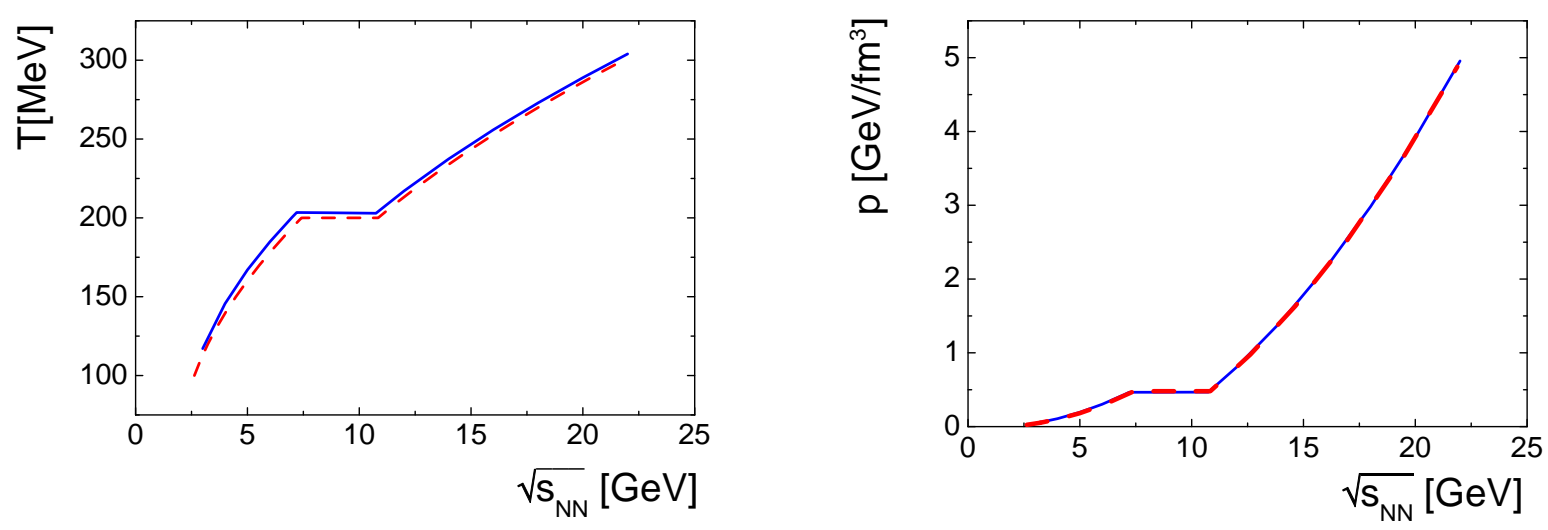

Figure 4: The CE temperature (left) and pressure (right) as function of collision energy are shown by the solid lines. The dashed lines correspond to the GCE result presented in Fig. 2.

The collision energy dependence of $T$ and $p$ obtained within the CE is shown by the solid lines in Fig. 4 left and right, respectively. The dashed lines in Fig. 4 correspond to the GCE results presented in Fig. 2. The CE and GCE curves are similar. A slightly larger value of $T$ in the CE than in the GCE is needed to compensate the CE suppression of energy density. Note that $\varepsilon$ as a function of $\sqrt{s_{N N}}$ is given by Eq. (3) and, thus, it is independent of the system size. The entropy density is given by Eq. (10) in terms of $p, \varepsilon$, and $T$. Therefore, the entropy density $s$ is weakly affected by the exact strangeness conservation imposed in the CE.

The Gibbs criterion (28) used in the CE is again equivalent to the maximum entropy condition. This is illustrated in Fig. 5 left, where the ratios $R_{i}=\left(s_{i} / s_{Q}\right)_{\mathrm{CE}}$ calculated in the CE are presented for entropies $s_{H}, s_{Q}$ and $s_{m i x}$ for $A_{p}=1$. 

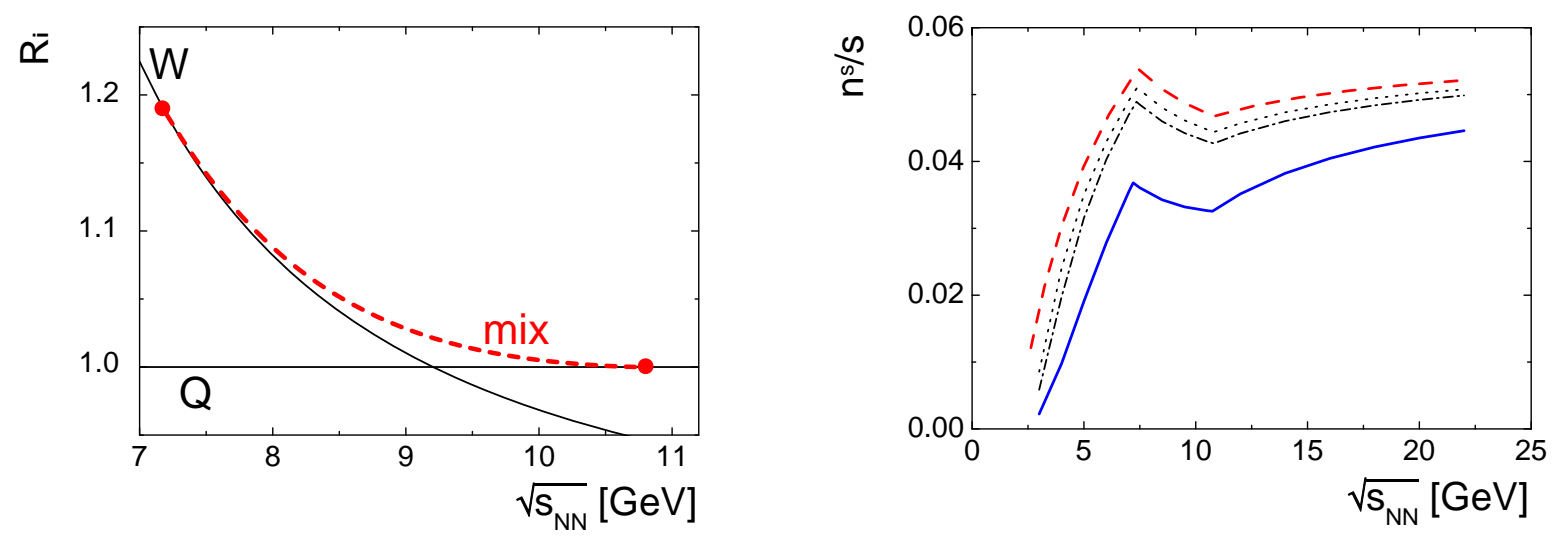

Figure 5: (a): The $\mathrm{CE}\left(A_{p}=1\right)$ ratio of entropy densities $s_{i} / s_{Q}$ with $i$ referring to the $\mathrm{W}$ (solid line), Q (horizontal solid line), and mixed (dashed line) phases as a function of the collision energy. The full circles correspond to the beginning and end of the mixed phase given by Eq. 29) and Eq. (30), respectively. (b): The CE strangeness to entropy ratio as a function of the collision energy. The solid line corresponds to $A_{p}=1$ and the dashed line to $A_{p} \gg 1$ which coincides with the GCE results presented in Fig. 3 (b). The dashed-dotted and dotted lines show the CE results for $A_{p}=3$ and 5 , respectively.

Figure 5 right presents energy dependence of the strangeness to entropy ratio, $n^{s} / s$, calculated within the $\mathrm{CE}$ for $A_{p}=1,3$ and 5 as well as the result for the $\operatorname{GCE}\left(A_{p} \gg 1\right)$.

In Fig. 6 the ratio

$$
r \equiv \frac{\left[n^{s} / s\right]_{\mathrm{CE}}}{\left[n^{s} / s\right]_{\mathrm{GCE}}}
$$

is shown as a function of $A_{p}$ at three collision energies in the vicinity of the transition region. It is seen that the CE suppression of the strangeness to entropy ratio depends strongly on the number of participants $2 A_{p}$. With increasing $A_{p}$ the CE suppression decreases. At $\sqrt{s_{N N}}>10 \mathrm{GeV}$ the suppression parameter (31) is close to unity already for $A_{p}>10$. The CE suppression increases with decreasing collision energy, when the total number of strange particles is small. This is indicated by the dotted line in Fig. 6 which is calculated for collisions at $\sqrt{s_{N N}}=5 \mathrm{GeV}$.

Finally, the strangeness to entropy ratio calculated for central $\mathrm{Pb}+\mathrm{Pb}$ collisions (the $\mathrm{GCE}$ result) and inelastic $\mathrm{p}+\mathrm{p}$ interactions (the $\mathrm{CE}$ with $A_{p}=1$ result) is plotted in Fig. 7 as 


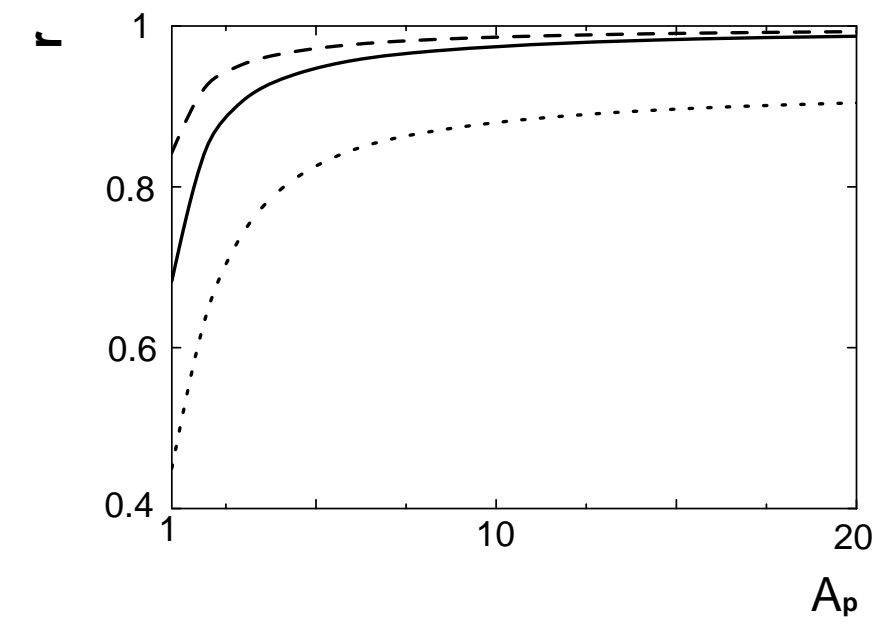

Figure 6: The CE strangeness to entropy ratio divided by the corresponding ratio in the GCE is shown as a function of $A_{p}$. The double ratio (Eq. (31)) is calculated at the beginning of the mixed phase $\sqrt{s_{N N}} \approx 7.3 \mathrm{GeV}$ (solid line), below the mixed phase region $\sqrt{s_{N N}}=5 \mathrm{GeV}$ (dotted line), and above the mixed phase region $\sqrt{s_{N N}}=20 \mathrm{GeV}$ (dashed line).

a function of collision energy up to the LHC energies. The left plot shows the two ratios separately, whereas the right one presents their ratio. The energy dependence predicted by the SMES is only qualitatively similar to the measured one (Fig. 1). Clearly the SMES, the simplest model of the onset of deconfinement, has to be significantly modified in order to reach a quantitative agreement with the data.

\section{SUMMARY}

This paper introduces the exact strangeness conservation in the Statistical Model of the Early Stage [3] of nucleus-nucleus collisions. This allows to calculate the energy dependence of the strangeness to entropy ratio for collisions of protons and small nuclei at the CERN SPS energies. The extension of the model is motivated by the recent results of the NA61/SHINE experiment at the CERN SPS on hadron production in inelastic $\mathrm{p}+\mathrm{p}$ interactions [10], which suggest that the deconfinement may take place also in this reaction. 

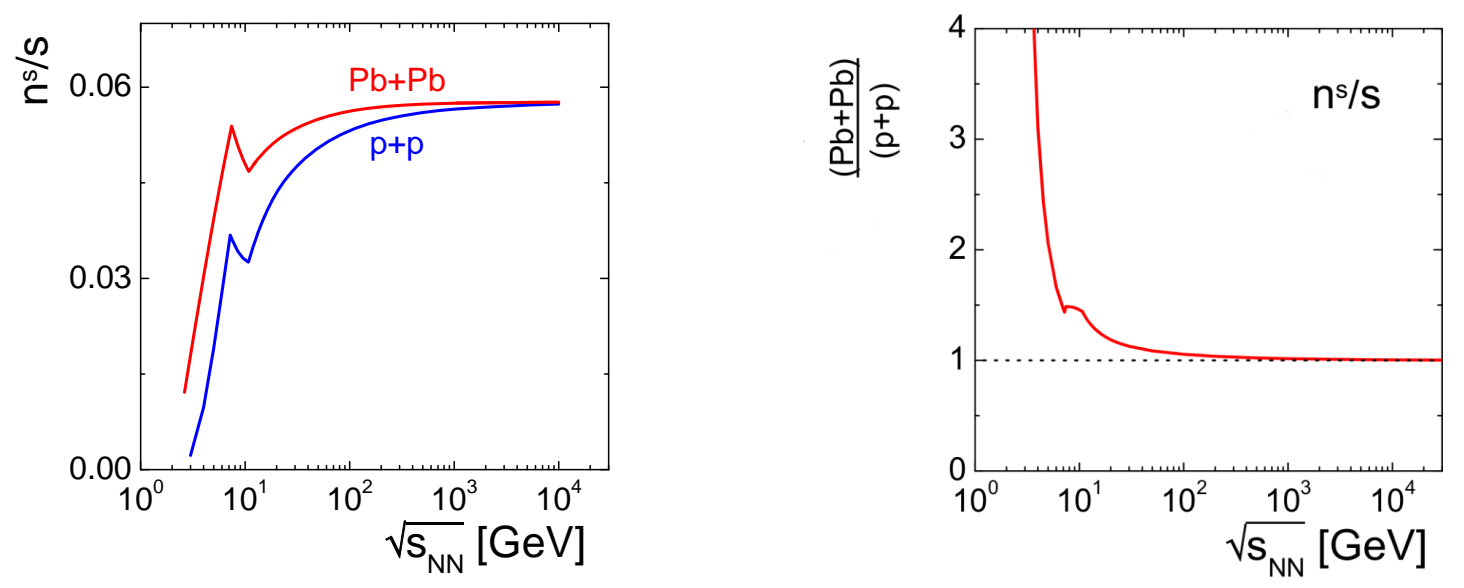

Figure 7: Left: Collision energy dependence of the strangeness to entropy ratio calculated within the SMES for central $\mathrm{Pb}+\mathrm{Pb}$ collisions (the GCE result) and inelastic $\mathrm{p}+\mathrm{p}$ interactions (the $\mathrm{CE}$ result for $\left.A_{p}=1\right)$. The ratio is plotted up to the LHC energies. Right: The ratio of strangeness to entropy ratios calculated for central $\mathrm{Pb}+\mathrm{Pb}$ collisions and inelastic $\mathrm{p}+\mathrm{p}$ interactions with the SMES as a function of collision energy.

The CE treatment of the strangeness production leads to the well known effect - the total number of strange and anti-strange particles is reduced in comparison to that obtained within the GCE at the same values of volume and energy density. However, the calculations show only small modifications of the system temperature, pressure, and entropy density. Thus, the strangeness to entropy ratio is significantly reduced in small systems. The smaller the collision energy, the smaller is the total number of strange particles, and, thus, the stronger is the $\mathrm{CE}$ strangeness suppression. In the region of the mixed phase, $\sqrt{s_{N N}}=7-11 \mathrm{GeV}$, the strangeness to entropy ratio in $\mathrm{p}+\mathrm{p}$ interactions is found to be approximately two times smaller than in central $\mathrm{Pb}+\mathrm{Pb}$ collisions. Note that the $\mathrm{CE}$ suppression becomes quite small already for central collisions of intermediate size nuclei and it is negligible for central $\mathrm{Pb}+\mathrm{Pb}$ collisions. The calculated collision energy dependence of the strangeness to entropy ratio in $\mathrm{p}+\mathrm{p}$ interactions is qualitatively similar to the one measured by the NA61/SHINE collaboration [9, 10] for the $K^{+}$to $\pi^{+}$ratio (see Fig. 1). However, a quantitative comparison between the model and the data requires further modifications of the model and thus being beyond the scope of this paper. 


\section{Acknowledgments}

We would like to thank Marysia Gazdzicka for corrections to the paper. This work was supported by the National Science Centre of Poland (grant UMO-2012/04/M/ST2/00816), the German Research Foundation (grant GA 1480/2-2) and and the National Academy of Sciences of Ukraine, Research Grant ZO-2-1/2015.

[1] W. Florkowski, Phenomenology of Ultra-Relativistic Heavy-Ion Collisions World Scientific, ISBN: 9814280666, 436 pages, 2010.

[2] S. V. Afanasev et al. [NA49 Collaboration], CERN-SPSC-2000-035, CERN-SPSLC-P-264-ADD-7.

[3] M. Gazdzicki and M. I. Gorenstein, Acta Phys. Pol. B30, 2705 (1999).

[4] C. Alt et al. [NA49 Collaboration], Phys. Rev. C 77, 024903 (2008).

[5] S. V. Afanasiev et al. [NA49 Collaboration], Phys. Rev. C 66, 054902 (2002).

[6] A. Rustamov, Central Eur. J. Phys. 10, 1267 (2012).

[7] M. Gazdzicki, M. I. Gorenstein, and P. Seyboth, Acta Phys. Polon. B 42, 307 (2011);

M. Gazdzicki, M. I. Gorenstein, and P. Seyboth, Int. J. Mod. Phys. E 23, 1430008 (2014).

[8] J. Baacke, Acta Phys. Polon. B 8, 625 (1977);

E. V. Shuryak, Phys. Rept. 61, 71 (1980);

J. Cleymans, R. V. Gavai and E. Suhonen, Phys. Rept. 130, 217 (1986).

[9] N. Abgrall et al. [NA61 Collaboration], Eur. Phys. J. C 74, 2794 (2014).

[10] N. Abgrall et al., CERN-SPSC-2014-031, SPSC-SR-145.

[11] L. D. Landau, Izv. Akad. Nauk Ser. Fiz. 17, 51 (1953); S. Z. Belenkij and L. D. Landau, Nuovo Cim. Suppl. 3S10, 15 (1956) [Usp. Fiz. Nauk 56, 309 (1955)].

[12] L. P. Csernai, J. I. Kapusta, and L. D. McLerran, Phys. Rev. Lett. 97, 152303 (2006).

[13] M. I. Gorenstein, M. Hauer, and O. N. Moroz, Phys. Rev. C 77, 024911 (2008).

[14] J. Rafelski and M. Danos, Phys. Lett. B 97, 279 (1980).

[15] J. Clymans, K. Redlich, and E. Suhonen, Z. Phys. C 51, 137 (1991).

[16] F. Becattini, Z. Phys. C 69, 485 (1996) and Nucl. Phys. Proc. Suppl. 92, 137 (2001); 
F. Becattini and U. Heinz, Z. Phys. C 76, 269 (1997).

[17] M.I. Gorenstein, M. Gaździcki, and W. Greiner, Phys. Lett. B 483, 60 (2000);

M.I. Gorenstein, A. P. Kostyuk, H. Stöcker, and W. Greiner, Phys. Lett. B 509, 277 (2001);

M.I. Gorenstein, W. Greiner, and A. Rustamov, Phys. Lett. B 731, 302 (2014). 